\title{
CARACTERIZAÇÃO ECOFISIOLÓGICA DE LINHAGENS DE MILHO SUBMETIDAS A BAIXA DISPONIBILIDADE HÍDRICA DURANTE O FLORESCIMENTO
}

\author{
PAULO CÉSAR MAGALHÃES ${ }^{1}$, THIAGO CORRÊA DE SOUZA², PAULO EMÍLIO \\ PEREIRA DE ALBUQUERQUE ${ }^{1}$, DÉCIO KARAM ${ }^{1}$, MARCELO MURAD MAGALHÃES ${ }^{2}$ \\ e FERNANDO RODRIGO DE OLIVEIRA CANTÃO ${ }^{1}$
}

${ }^{1}$ Embrapa Milho e Sorgo, Sete Lagoas, MG, Brasil, Caixa Postal 151, CEP 35701-970,pcesar@ cnpms.embrapa.br

${ }^{2}$ Departamento de Biologia, Setor de Fisiologia Vegetal, Campus Universitário, Lavras, $M G$, Brasil, caixa postal 37,CEP37200-000,thiagonepre@hotmail.com

Revista Brasileira de Milho e Sorgo, v.8, n.3, p. 223-232, 2009

RESUMO - O estudo de cultivares tolerantes a limitações hídricas é uma alternativa sustentável para mitigar os impactos negativos das mudanças climáticas globais. Este trabalho foi realizado com o objetivo de caracterizar linhagens de milho contrastantes quanto a resistência a seca no estádio de florescimento, baseando-se nas trocas gasosas e fluorescência da clorofila, em casa-de-vegetação. Foram avaliadas três linhagens de milho, sendo duas tolerantes (L 31.2.1.2 e L 29.1.1) e uma sensível (L 2.3.2.1) a deficiência hídrica. No pré-florescimento, dois tratamentos hídricos, sem (SD) e com deficiência hídrica (CD), foram impostos, e após cinco dias de imposição do estresse, foram avaliadas a taxa de fotossíntese foliar (A), a condutância estomática (gs), a transpiração foliar (T), a eficiência intrínseca do uso da água (EIUA, A/gs), o teor relativo de clorofila (SPAD) e parâmetros da fluorescência da clorofila (Fv/Fm, Fo/ FM, Fv/Fo). As linhagens tolerantes a seca apresentaram maior eficiência no uso da água em comparação com a linhagem sensível. A linhagem L 29.1.1 apresentou maior eficiência no fotossistema II (Fv/Fm) do que as linhagens L 2.3.2.1 e L 31.2.1.2. Podese concluir que existem diferenças ecofisiológicas nas trocas gasosas e na fluorescência da clorofila, que caracterizam as linhagens tolerantes à seca em relação às linhagens sensíveis. Os resultados desta pesquisa fornecerão subsídios para os melhoristas selecionarem material tolerante a seca.

Palavras-chaves: trocas gasosas, Zea mays L., seca, fluorescência da clorofila 


\title{
ECOPHYSIOLOGICAL CHARACTERIZATION OF MAIZE LINES SUBMITTED TO LOW WATER AVAILABILITY DURING FLOWERING
}

\begin{abstract}
The study of cultivars tolerant to water limitation is a sustainable alternative to mitigate the negative impacts of global climatic changes. This study was performed with the objective of characterizing maize lines exhibiting contrasting drought resistance at flowering stage, using gaseous exchange and chlorophyll fluorescence, under greenhouse conditions. Three maize lines were evaluated, being two tolerant (L 31.2.1.2 and L 29.1.1) and one sensitive (L2.3.2.1) to water deficit. At pre-flowering growth stage, two water treatments were imposed: without stress (WS) and with water deficit (WD). Five days after imposing the water stress the following characteristics were evaluated: photosynthesis rate (A), stomatal conductance (gs), leaf transpiration (T), intrinsic water use efficiency (iWUE, A/gs), relative chlorophyll content (SPAD) and chlorophyll fluorescence parameters (Fv/Fm; Fo/FM; Fv/Fo). The drought tolerant lines showed greater efficiency in water use, compared to the sensitive line. The line L 29.1.1 presented greater photosystem II efficiency (Fv/Fm) than lines L 2.3.2.1 and L 31.1.2. Based on the results it can be concluded that there are ecophysiological differences on gaseous exchange and chlorophyll fluorescence, which characterize the drought tolerant lines, when compared to the sensitive one. The results obtained in this research will be useful for plant breeders to select drought tolerant genotypes.
\end{abstract}

Key words: gaseous exchange, Zea Mays L., drought, chlorophyll fluorescence

O estresse pela baixa disponibilidade hídrica (seca) é um dos principais problemas da agricultura, e a habilidade das plantas para resistir a tal estresse é de suma importância para o desenvolvimento do agronegócio de qualquer país (Shao et al., 2008). Estudos de tolerância a seca envolvendo o milho podem trazer melhorias no crescimento e no rendimento da cultura em regiões com limitação hídrica (Li et al., 2009), já que o milho é conhecido pela sua alta sensibilidade a esse estresse (Vamerali et al., 2003; Welcker et al., 2007). Além disso, material tolerante a esse estresse abiótico agrega valor à semente para a comercialização.

Grandes avanços em estudos sobre melhoramento do milho para seca têm trazido resultados satisfatórios, gerando genótipos tolerantes (Campos et al., 2004; Monneveux et al., 2006). Contudo, pouco se sabe sobre os mecanismos fisiológicos para a tolerância a seca (Banziger et al., 2002). Nesse sentido, a caracterização dos materiais genéticos, assim 
como a elucidação dos possíveis mecanismos responsáveis pelo comportamento diferencial de genótipos sob condição de estresse, pode fornecer parâmetros que auxiliarão na seleção de novos materiais genéticos, com grande probabilidade de redução do tempo de liberação do produto para comercialização.

A avaliação da fotossíntese em estudos com a planta de milho, em diferentes condições ambientais, é uma prática importante para uma seleção mais “pontual” de genótipos, objetivando obter altas produtividades em meio à limitação climática (Kim et al., 2006). Juntamente com a fotossíntese e outros parâmetros de trocas gasosas, a fluorescência da clorofila também pode ajudar no entendimento fisiológico do processo em condições de seca, sendo que os padrões de fluorescência nessas condições são bem estabelecidos (Long \& Bernacchi 2003). A eficiência do fotossistema II revela o nível de excitação da energia no sistema de pigmentos envolvidos na fotossíntese, e tem-se constituído uma potente ferramenta de seleção de plantas de milho tolerantes a condições adversas, como a seca (Durães et al., 2005).

As respostas fisiológicas na tolerância a seca podem variar de acordo com a severidade e a duração de imposição do estresse, estádio fenológico e material genético (Durães et al., 2002; Shao et al., 2008). Com relação ao estádio fenológico, de acordo com Edmeades et al (2000), o milho é particularmente muito sensível no estádio de florescimento. Seca durante esse período leva a um aumento no intervalo entre florescimento masculino e feminino (IFMF), que é negativamente correlacionado com a produção (Duvick, 2005).

Tendo conhecimento da variabilidade genética para tolerância a seca, este trabalho visou caracterizar linhagens de milho contrastantes quanto a resistência a seca no estádio de florescimento pleno, por meio das trocas gasosas e fluorescência da clorofila.

\section{Material e Métodos}

O experimento foi realizado na Embrapa Milho e Sorgo, situado no município de Sete Lagoas, MG, Brasil. Foram avaliadas três linhagens de milho com background genético e origens distintas, sendo duas tolerantes (L 31.2.1.2 e L 29.1.1) e uma sensível (L 2.3.2.1) a seca, oriundas do Programa de Melhoramento da Embrapa Milho e Sorgo. O ensaio foi conduzido em casa-de-vegetação, com duas plantas por vaso de 20 litros, com solo tipo Latossolo Vermelho Distrófico Típico. As adubações de plantio e cobertura foram realizadas de acordo com a recomendação para a cultura no Estado de Minas Gerais (Ribeiro et al., 1999). $\mathrm{O}$ delineamento experimental utilizado foi o inteiramente casualizado, com seis repetições.

$\mathrm{O}$ teor de água no solo foi monitorado diariamente nos períodos da manhã e da tarde (9 e 15 horas), com o auxílio de um sensor de umidade modelo GBReader, N 1535 (Measurement Engineering, Austrália), instalado no centro dos vasos, na profundidade de $20 \mathrm{~cm}$. 
A reposição hídrica foi realizada com base nas leituras obtidas com o sensor e a água foi reposta até a capacidade de campo (CC). Esses cálculos foram realizados com o auxílio de uma planilha eletrônica, feita em função da curva de retenção de água do solo.

No pré-florescimento, foram impostos dois tratamentos hídricos: sem (SD) e com deficiência hídrica (CD). No primeiro, a reposição hídrica foi realizada diariamente até o solo atingir a umidade na $\mathrm{CC}$, enquanto que, no segundo tratamento, a indução do estresse hídrico foi realizada diariamente aplicando-se $50 \%$ da água total disponível, ou seja, até o potencial da água no solo atingir aproximadamente $-70 \mathrm{kPa}$, cujo valor corresponde ao solo especificado. Esse estresse foi mantido por sete dias. As avaliações foram realizadas cinco dias após a imposição do estresse hídrico, no momento em que as plantas se encontravam no florescimento pleno. Foram avaliadas a taxa de fotossíntese foliar (A), condutância estomática (gs), transpiração foliar (T) e eficiência intrínseca do uso da água (EIUA, A/gs), utilizando um sistema portátil de trocas gasosas IRGA LI 6400 (Portable Photosynthesis System LICOR, Nebraska, USA). As medidas foram feitas em uma área foliar de $6 \mathrm{~cm}^{2}$, com um fluxo de ar na câmara de $1200 \mu \mathrm{mol} \mathrm{s}{ }^{-1}$ e uma intensidade de radiação fotossinteticamente ativa de 1.500 $\mu \mathrm{mol} \mathrm{m} \mathrm{m}^{-2} \mathrm{~s}^{-1}$. Após cinco dias de imposição do estresse hídrico, foi estimado o teor relativo de clorofila por leituras no SPAD ("Soil plant analysis development", Minolta SPAD 502 Osaka, Japan). Em folhas adaptadas ao escuro, foi determinada a eficiência quântica potencial $(\mathrm{Fv} / \mathrm{Fm})$ do fotosistema II (FSII), o rendimento quântico basal dos processos fotoquímicos no FSII ( $\mathrm{Fo} / \mathrm{Fm})$ e a eficiência fotoquímica no FSII $(\mathrm{Fv} / \mathrm{Fo})$, por meio de um fluorímetro (Plant Efficiency Analyser, Hansatech Instruments King's Lynn, UK). O acondicionamento das folhas foi realizado com a ajuda de clipes foliares, e a intensidade de luz no sensor foi de $60 \%$ da capacidade total do aparelho, durante cinco segundos em cada leitura. Todas as leituras foram realizadas na folha bandeira, no período da manhã, entre as 8 e as 10 horas.

Para a análise estatística dos resultados, utilizou-se a análise de variância e o teste de comparação de médias Skott-Knott, a 5\% de probabilidade, no programa estatístico SISVAR versão 4.3 (Ferreira, 2000).

\section{Resultados e Discussão}

Os resultados relativos à fluorescência da clorofila indicaram a superioridade da linhagem L 29.1.1 na condição de estresse hídrico (CD), com valores maiores de $\mathrm{Fv} / \mathrm{Fm}$ e $\mathrm{Fv} / \mathrm{Fo}$, $(0,634$ e 1,783 , respectivamente) em relação às linhagens L 2.3.2.1 e L 31.2.1.2 (Tabela 1). Por outro lado, essas duas linhagens tiveram valores superiores a L 29.1.1 na relação Fo/Fm. $\mathrm{Na}$ condição sem estresse hídrico (SD), não houve diferença entre as linhagens. Na média dos ambientes, as plantas sem deficiência 
TABELA 1 - Eficiência quântica potencial (Fv/Fm), rendimento quântico basal dos processos fotoquímicos (Fo/Fm), eficiência fotoquímica no fotosistema II (Fv/Fo), em plantas de milho no pré-florescimento, sem (SD) e com (CD) deficiência hídrica. Sete Lagoas, MG. 2008.

\begin{tabular}{|c|c|c|c|c|c|c|}
\hline \multicolumn{7}{|c|}{ Características ecofisiológicas ${ }^{1}$} \\
\hline \multirow[t]{2}{*}{ Genótipos } & \multicolumn{2}{|c|}{ Fv/Fm } & \multicolumn{2}{|c|}{ Fo/Fm } & \multicolumn{2}{|c|}{ Fv/Fo } \\
\hline & SD & $\mathrm{CD}$ & $\mathrm{SD}$ & $\mathrm{CD}$ & $\mathrm{SD}$ & $\mathrm{CD}$ \\
\hline $\mathrm{L} 2.3 .2 .1$ & $0,682 \mathrm{~A}$ & $0,546 \mathrm{~A}$ & $0,314 \mathrm{~A}$ & $0,453 \mathrm{~B}$ & $2,203 \mathrm{~A}$ & $1,223 \mathrm{~A}$ \\
\hline L 29.1.1 & 0,692 A & $0,634 \mathrm{~B}$ & $0,307 \mathrm{~A}$ & $0,359 \mathrm{~A}$ & $2,346 \mathrm{~A}$ & $1,783 \mathrm{~B}$ \\
\hline L 31.2.1.2 & $0,679 \mathrm{~A}$ & $0,536 \mathrm{~A}$ & $0,317 \mathrm{~A}$ & $0,470 \mathrm{~B}$ & $2,154 \mathrm{~A}$ & $1,145 \mathrm{~A}$ \\
\hline Média ambientes & $0,684 \mathrm{~b}$ & $0,572 \mathrm{~b}$ & $0,313 \mathrm{a}$ & $0,427 \mathrm{~b}$ & $2,234 \mathrm{~b}$ & $1,383 \mathrm{a}$ \\
\hline CV (\%) & \multicolumn{2}{|c|}{10,40} & \multicolumn{2}{|c|}{16,93} & \multicolumn{2}{|c|}{19,81} \\
\hline Média geral & \multicolumn{2}{|c|}{0,628} & \multicolumn{2}{|c|}{0,37} & \multicolumn{2}{|c|}{1,8} \\
\hline
\end{tabular}

hídrica foram significativamente superiores às plantas com deficiência em todos os parâmetros relativos à fluorescência da clorofila.

Para avaliação do teor relativo de clorofila, utilizando o clorofilômetro, não foram detectadas diferenças estatisticamente significativas entre as linhagens submetidas a condição com e sem deficiência hídrica. No entanto, a media dos ambientes revelou superioridade significativa do ambiente sem deficiência hídrica (SD) (Tabela 2). Com relação à taxa fotossintética (A), não se observaram diferenças significativas nas linhagens submetidas a deficiência hídrica (CD), enquanto que, nas plantas sem deficiência hídrica (SD), houve predominância significativa da linhagem L 29.1.1 sobre as demais. Na média dos ambientes, houve diferença na fotossíntese, sendo que o ambiente sem estresse (SD) foi significativamente maior (Tabela 2).

Para a condutância estomática (gs), não foi possível detectar diferenças estatisticamente significativas entre as linhagens, embora o ambiente sem deficiência hídrica (SD), na média, tenha superado o ambiente com deficiência hídrica (Tabela 3). A transpiração (T) no ambiente submetido a estresse hídrico não resultou em diferenças entre as linhagens, enquanto que, nas plantas sem deficiência hídrica, a L 2.3.2.1 foi inferior em relação às demais. Na média dos ambientes, as plantas sem deficiência tiveram, significativamente, uma transpiração maior do que as plantas com 
TABELA 2 - Teor relativo de clorofila (SPAD) e taxa de fotossíntese foliar $(A)$, em plantas de milho no pré-florescimento, sem (SD) e com (CD) deficiência hídrica. Sete Lagoas, MG. 2008.

\begin{tabular}{|c|c|c|c|c|}
\hline \multicolumn{5}{|c|}{ Características ecofisiológicas ${ }^{1}$} \\
\hline \multirow[t]{2}{*}{ Genótipos } & \multicolumn{2}{|c|}{ Clorofila - SPAD } & \multicolumn{2}{|c|}{ A $\left(\mu\right.$ moles de $\left.\mathrm{CO}_{2} \mathrm{~m}^{-2} \mathrm{~s}^{-1}\right)$} \\
\hline & SD & $\mathrm{CD}$ & SD & $\mathrm{CD}$ \\
\hline L 2.3.2.1 & $39,93 \mathrm{~A}$ & $32,41 \mathrm{~A}$ & $32,26 \mathrm{~A}$ & $10,07 \mathrm{~A}$ \\
\hline L 29.1.1 & $40,30 \mathrm{~A}$ & $35,11 \mathrm{~A}$ & $41,18 \mathrm{~B}$ & $11,59 \mathrm{~A}$ \\
\hline L 31.2.1.2 & $46,85 \mathrm{~A}$ & $38,45 \mathrm{~A}$ & $38,58 \mathrm{~A}$ & $9,18 \mathrm{~A}$ \\
\hline Média ambientes & $42,36 \mathrm{~b}$ & $35,32 \mathrm{a}$ & $37,34 \mathrm{~b}$ & $10,28 \mathrm{a}$ \\
\hline $\mathrm{CV}(\%)$ & \multicolumn{2}{|c|}{12,26} & \multicolumn{2}{|c|}{20,97} \\
\hline Média geral & \multicolumn{2}{|c|}{39,46} & \multicolumn{2}{|c|}{23,81} \\
\hline
\end{tabular}

${ }^{1}$ Médias seguidas pelas mesmas letras maiúsculas, na coluna, e minúsculas, na linha, não diferem entre si pelo teste Scott-Knott, a 5\% de probabilidade.

deficiência (Tabela 3). Na eficiência intrínseca do uso da água(EIUA), também houve diferença significativa nas médias dos ambientes, observando-se valores maiores no ambiente com deficiência hídrica. Para as plantas sem deficiência hídrica (SD), não houve diferenças entre as linhagens, porém, nas plantas com deficiência hídrica (CD), as linhagens tolerantes a seca (L 29.1.1 e L 31.2.1.2) apresentaram valores significativamente maiores na EIUA, com relação à linhagem sensível (L 2.3.2.1) (Tabela 3).

O déficit hídrico como um fator de estresse pode causar ação inibitória na fase bioquímica da fotossíntese (Xu et al., 2008). Sabendo-se que o fotossistema II é responsável pelo fornecimento de energia para a fotossíntese, a avaliação de sua eficiência pode tornar-se um indicador da tolerância em plantas sob estresse de seca. Portanto, neste trabalho, a avaliação da fluorescência da clorofila foi importante para a identificação das linhagens de milho menos suscetíveis a danos causados pelo estresse hídrico, sendo que a linhagem L 29.1.1 foi a que apresentou maior eficiência fotoquímica no FSII (Fv/Fm). Este é o principal parâmetro utilizado para a avaliação desses danos no sistema fotossintético, uma vez que a eficiência fotoquímica do FSII indica quando todos os centros de reação estão abertos (Baker \& Rosenqvst, 2004). Embora a razão Fv/Fm normalmente decresça em plantas submetidas a algum tipo de estresse (Krause \& Weis, 1991), não foi observada influência do 
déficit hídrico nessa relação em trigo (Hura et al, 2007), diferentemente de Xu et al. (2009), que detectaram mudanças na relação Fv/Fm em gramíneas (Leymus chinensis (Trin.) Tzvel.) sob deficiência hídrica. Já Rahman et al. (2004), estudando duas cultivares de milho, concluíram que o decréscimo no crescimento desses genótipos sob seca foi devido à diminuição da eficiência fotoquímica do fotossistema II (Fv/ Fm).

No presente trabalho, a imposição de cinco dias de estresse (deficiência hídrica) não foi capaz de alterar a concentração da clorofila. Em muitos casos, inclusive no milho, a deficiência hídrica induz a uma redução da clorofila (Grzesiak, et al., 2007). O teor de clorofila pode ser considerado como uma característica secundária. Em milho, seu aumento pode estar associado com o rendimento da cultura (Betrán et al., 2003).

Nos dois ambientes estudados, com e sem deficiência hídrica, não ocorreram mudanças significativas entre as linhagens nos parâmetros de taxa de fotossíntese, condutância estomática e transpiração. Entretanto, quanto à eficiência intrínseca do uso da água (EIUA), as linhagens tolerantes (L 29.1.1 e L 31.2.1.2) sobressaíramse em relação à linhagem sensível (L 2.3.2.1), no ambiente com deficiência hídrica, mostrandose mais tolerantes ao estresse hídrico, devido à economia de água durante as trocas gasosas. Essa economia de água nas linhagens tolerantes pode ter ocorrido devido à menor condutância estomática (gs), porém não foram detectadas

TABELA 3 - Condutância estomática (gs), transpiração ( $\mathrm{T}$ ) e eficiência intrínseca do uso da água (EIUA ) em plantas de milho no pré-florescimento, sem (SD) e com (CD) deficiência hídrica. Sete Lagoas, MG. 2008.

\begin{tabular}{|c|c|c|c|c|c|c|}
\hline \multicolumn{7}{|c|}{ Características ecofisiológicas ${ }^{1}$} \\
\hline \multirow[t]{2}{*}{ Genótipos } & \multicolumn{2}{|c|}{ gs $\left(\mathrm{mmol} \mathrm{m} \mathrm{m}^{-2} \mathrm{~s}^{-1}\right)$} & \multicolumn{2}{|c|}{$T\left(\mu g \mathbf{c m}^{-2} \mathbf{s}^{-1}\right)$} & \multicolumn{2}{|c|}{ EIUA $\left[\left(\mu \mathrm{mol} \mathrm{CO}\left(\operatorname{mol~H}_{2} \mathrm{O}\right)^{-1}\right]\right.$} \\
\hline & SD & CD & SD & $\overline{C D}$ & SD & CD \\
\hline L 2.3.2.1 & $0,210 \mathrm{~A}$ & $0,049 \mathrm{~A}$ & $5,496 \mathrm{~A}$ & $1,299 \mathrm{~A}$ & $155,0 \mathrm{~A}$ & $206,2 \mathrm{~A}$ \\
\hline L 29.1.1 & $0,268 \mathrm{~A}$ & $0,038 \mathrm{~A}$ & $9,208 \mathrm{~B}$ & $1,306 \mathrm{~A}$ & $157,4 \mathrm{~A}$ & 404,4 B \\
\hline L 31.2.1.2 & $0,248 \mathrm{~A}$ & $0,019 \mathrm{~A}$ & $9,325 \mathrm{~B}$ & $0,938 \mathrm{~A}$ & $167,6 \mathrm{~A}$ & $475,6 \mathrm{~B}$ \\
\hline Média ambientes & $0,242 \mathrm{~b}$ & $0,035 \mathrm{a}$ & $8,009 \mathrm{~b}$ & $1,181 \mathrm{a}$ & $160,0 \mathrm{a}$ & $362,0 \mathrm{~b}$ \\
\hline $\mathrm{CV}(\%)$ & \multicolumn{2}{|c|}{37,26} & \multicolumn{2}{|c|}{31,66} & \multicolumn{2}{|c|}{26,00} \\
\hline Média geral & \multicolumn{2}{|c|}{0,139} & \multicolumn{2}{|c|}{4,59} & \multicolumn{2}{|r|}{261,0} \\
\hline
\end{tabular}

${ }^{1}$ Médias seguidas pelas mesmas letras maiúsculas, na coluna, e minúsculas, na linha, não diferem entre si pelo teste Scott-Knott, a $5 \%$ de probabilidade. 
diferenças estatísticas significativas na condutância.

A fotossíntese sob estresse hídrico tem sido pouco estudada em plantas tipo $\mathrm{C}_{4}$, como o milho (Ghannoum, 2009). Sob uma deficiência hídrica moderada, o milho apresenta decréscimo da fotossíntese devido à diminuição da condutância estomática, levando ao fechamento dos estômatos e à redução da transpiração. Contudo, sob um estresse severo e prolongado, limitações não estomáticas (bioquímicas) começam a ocorrer (Grzesiak et al.,2006; 2007). Diminuição da condutância e fotossíntese foi observada por $\mathrm{Xu}$ et al. (2008), em uma variedade de milho chinesa sob deficiência hídrica. Hund et al (2009) avaliaram a tolerância de híbridos ao déficit hídrico e observaram aumento na eficiência intrínseca do uso da água (EIUA). Segundo esses autores, o aumento na EIUA é um importante fator para identificar híbridos adaptados ao estresse.

\section{Conclusões}

Existem diferenças nas trocas gasosas e na fluorescência da clorofila que caracterizam as linhagens tolerantes a seca em relação às linhagens sensíveis.

As linhagens tolerantes a seca apresentaram maior eficiência no uso da água, em comparação com a linhagem sensível.

A linhagem L 29.1.1 apresentou maior eficiência no fotossistema II do que as linhagens L 2.3.2.1 e L 31.2.1.2.

\section{Literatura Citada}

BAKER, N. R.; ROSENQVST, E. Applications of chlorophyll fluorescence can improve crop production strategies: an examination of future possibilities. Journal of Experimental Botany, London, v. 55, p. 1607-1621, 2004.

BANZIGER, M.; EDMEADES, G. O.; LAFITTE, H. R. Physiological mechanisms contributing to the increased $\mathrm{N}$ stress tolerance of tropical maize selected for drought tolerance. Field Crops Research, Amsterdam, v. 75, p. 223-233, 2002.

BETRÁN, F. J.; BECK, D.; BANZIGER, M.; EDMEADES, G. O. Secondary traits in parental inbreds and hybrids under stress and non-stress environments in tropical maize. Field Crops Research, Amsterdam, v. 83, p. 51-65, 2003.

CAMPOS; H.; COOPER; M.; HABBEN, J. E.; EDMEADES, G. O.; SCHUSSLER, J. R. Improving drought tolerance in maize: a view from industry. Field Crops Research, Amsterdam, v. 90, p. 19-34, 2004.

DURÃES, F. O. M.; MAGALHÃES, P. C.; GAMA, E. E. G.; OliveIRA, A. C. Caracterização fenotípica de linhagens de milho quanto ao rendimento e à eficiência fotossintética. Revista Brasileira de Milho e Sorgo, Sete Lagoas, v. 4, n. 3, p 355-361, 2005.

DURÃES, F. O. M.; MAGALHÃES, P. C.; OLIVEIRA, A. C. de. Índice de colheita genético e as possibilidades da genética fisiológica para melhoramento do rendimento de milho. Revista Brasileira de Milho e Sorgo, Sete Lagoas, v. 1, n. 1, p. 33-40, 2002. 
DUVICK, D. N. The contribution of breeding to yield advances in maize (Zea mays L.). Advances in Agronomy, New York, v. 86, p. 83-145, 2005.

EDMEADES, G. O.; BOLANOS, J.; ELINGE, A.; RIBAUT, J. M.; BÄNZIGER, M.; WESTGATE, M. E.; The role and regulation of the anthesissilking interval in maize. In: WEStGate, M. E.; BOOTE, K. J. (Ed.). Physiology and modeling Kernel set in Maize. Madison: CSSA, 2000. p. 43-73. (CSSA Special Publication, 29).

FERREIRA, D. F. Manual do sistema Sisvar para análises estatísticas. Lavras: UFLA, 2000. 66 p.

GHANNOUM, O. $\mathrm{C}_{4}$ photosynthesis and water stress. Annals of Botany, London, v. 103, p. 635-644, 2009.

GRZESIAK, M. T.; GRZESIAK, S.; SKOCZOWSKI, A. Changes of leaf water potential and gas exchange during and after drought in triticale and maize genotypes differing in drought tolerance. Photosynthetica, Prague, v. 44, p. 561-568, 2006.

GRZESIAK, M. T.; RZEPKA, A.; HURA, T.; HURA, K.; SKOCZOWSKI, A. Changes in response to drought stress of triticale and maize genotypes differing in drought tolerance. Photosynthetica, Prague, v. 45, p. 280-287, 2007.

HUND, A.; RUYA, N.; LIEDGENS, M. Rooting depth and water use efficiency of tropical maize inbred lines, differing in drought tolerance. Plant and Soil, The Hague, v. 318, p. 311-325, 2009.
HURA, T.; GRZESIAK, S.; HURA, K.; THIEMT, E.; TOKARZ, K.; DZONY, M. W. Physiological and biochemical tools useful in drought-tolerance detection in genotypes of winter triticale: accumulation of ferulic acid correlates with drought tolerance. Annals of Botany, London, v. 100, p.767-775, 2007.

KIM, S. H.; SICHER, R. C.; BAE, H.; GITZ, D. C.; BAKER, J. T.; TIMLIN, D.; REDDY, V. R. Canopy photosynthesis, evapotranspiration, leaf nitrogen, and transcription profiles of maize in response to $\mathrm{CO} 2$ enrichment. Global Change Biology, Oxford, v. 12, p. 588-600, 2006.

KRAUSE, G. H.; WEIS, E. Chlorophyll fluorescence and photosynthesis: the basics. Annual Review of Plant Physiology and Plant Molecular Biology, Palo Alto, v. 42, p. 313349, 1991.

LI; Y.; SPERRY; J. S.; SHAO, M.; Hydraulic conductance and vulnerability to cavitation in corn (Zea mays L.) hybrids of differing drought resistance. Environmental and Experimental Botany, Oxford, v. 66, p. 341-346, 2009.

LONG, S. P.; BERNACCHI, C. J. Gas exchange measurements, what they can tell us about the underlying limitations to photosynthesis? Procedures and sources of error. Journal of Experimental Botany, London, v. 54, p. 23932401, 2003.

MONNEVEUX, P.; C.; SANCHEZ, C.; BECK, D.; Edmeades, G. O. Drought Tolerance Improvement in Tropical Maize Source Populations: Evidence of Progress. Crop Science, Madison, v. 46, p. 180-191, 2006.

RAHMAN, M. U.; GUL, S.;AHMADI, I. Effects of water stress on growth and photosynthetic 
pigments of corn (Zea mays L.) Cultivars. International Journal of Agriculture and Biology, Faisalbad, v. 6, n. 4, p. 652-655, 2004.

RIBEIRO, A. C.; GUIMARÃES, P. T. G.; ALVAREZ V.; V. H. (Ed.). Recomendação para o uso de corretivos e fertilizantes em Minas Gerais: 5a. aproximação. Viçosa, M: Comissão de Fertilidade do Solo do Estado de Minas Gerais, 1999. 359 p.

SHAO, H.; CHU, L.; JALEEL, C. A.; ZHAO, C.; Water-deficit stress induced anatomical changes in higher plants. Comptes Rendus Biologies, Paris, v. 331, p. 215-225, 2008.

VAMERALI, T.; SACCOMANI, M.; BONA, S.; MOSCA, G.; Guarise, M.; Ganis, A. A comparison of root characteristics in relation to nutrient and water stress in two maize hybrids. Plant and Soil, The Hague, v. 255, p.157-167, 2003.
XU, Z.; ZHOU, G.; SHIMIZU, H. Are plant growth and photosynthesis limited by predrought following rewatering in grass? Journal of Experimental Botany, London, v. 60, p. 3737-3749, 2009.

XU, Z. Z.; ZHOU, G. S.; WANG, Y. L.; HAN, G. X.; LI, Y. J. Changes in chlorophyll fluorescence in maize plants with imposed rapid dehydration at different leaf ages. Journal Plant Growth Regulation, Berlin, v. 27, p. 83-92, 2008.

WELCKER, C.; BOUSSUGE, B.; BENCIVENNI, C.; RIBAUT, M.; TARDIEU, F. Are source and sink strengths genetically linked in maize plants subjected to water deficit? A QTL study of the responses of leaf growth and of Anthesis-Silking Interval to water deficit. Journal of Experimental Botany, London, v. 58, p. 339-349, 2007. 